\title{
On cohomological obstructions for the existence of log-symplectic structures
}

\author{
IOAN MĂRCUT, AND BORIS OSORNO TORRES
}

\begin{abstract}
We prove that a compact log-symplectic manifold has a class in the second cohomology group whose powers, except maybe for the top, are nontrivial. This result gives cohomological obstructions for the existence of log-symplectic structures similar to those in symplectic geometry.
\end{abstract}

A Poisson structure $\pi$ on a smooth manifold $M$ of dimension $2 n$ is called a log-symplectic structure if the map

$$
\wedge^{n} \pi: M \longrightarrow \bigwedge^{2 n} T M, \quad x \mapsto \wedge^{n} \pi(x)
$$

is transverse to the zero section.

These structures were initially studied in the framework of deformation quantization in [4], where they are called b-symplectic structures. Later, their complex analogue was considered in [1], where they were first given the name log symplectic. In the context of Poisson geometry, this class of Poisson structures was introduced on two-dimensional surfaces in [5] (under the name of topologically stable Poisson structures) where a complete classification was obtained. In higher dimensions, a systematic investigation of the geometric properties of log-symplectic structures appeared in [3]. Their integrations by symplectic groupoids were studied in [2].

Our interest in log-symplectic structures comes from the fact that these can be used to construct regular corank-one Poisson structures. First, the singular locus of a log-symplectic structure $Z:=\left(\wedge^{n} \pi\right)^{-1}(0)$, if nonempty, carries a regular corank-one Poisson structure with a very special property: it has a transverse Poisson vector field [3]. Secondly, a log-symplectic structure can be used to construct a regular corank-one Poisson on $M \times S^{1}$, simply given by

$$
\pi+X \wedge \frac{\partial}{\partial \theta}
$$


where $X$ is a representative of the modular vector field of $(M, \pi)$. However, our result excludes the possibility of using this procedure to construct corank-one Poisson structures in some interesting examples, e.g., on $S^{4} \times S^{1}$.

Our result is the following:

Theorem. Let $\left(M^{2 n}, \pi\right)$ be a compact log-symplectic manifold. Then there exists a class $c \in H^{2}(M)$ such that $c^{n-1} \in H^{2 n-2}(M)$ is nonzero.

Proof. Denote by $Z:=\left(\wedge^{n} \pi\right)^{-1}(0)$ the singular locus of $\pi$. If $Z=\emptyset$, we can apply the usual argument from symplectic geometry. Assume that $Z \neq \emptyset$.

We first assume that $M$ is orientable. Let $\mu$ be a volume form on $M$ and denote by $t:=\left\langle\pi^{n}, \mu\right\rangle$. The singular locus becomes $Z=\{t=0\}$. The log-symplectic condition implies that $t$ is a submersion along $Z$, so we can find a retraction $r: U \rightarrow Z$, where $U$ is an open around $Z$, such that $(r, t)$ : $U \stackrel{\sim}{\longrightarrow} Z \times(-\delta, \delta)$ is a diffeomorphism. Since $Z$ is a Poisson submanifold (it is fixed by all Poisson automorphisms, hence all Hamiltonians are tangent to $Z$ ), in this open set $U$ we can write

$$
\left.\pi\right|_{U}=t \partial / \partial t \wedge X_{t}+w_{t}
$$

for a vector field $X_{t}$ and a bivector $w_{t}$ on $Z$, both depending smoothly on $t$. Since $(1 / t) \pi^{n}=n \partial / \partial t \wedge X_{t} \wedge w_{t}^{n-1}$ is nowhere vanishing, we have that the bivector $\partial / \partial t \wedge X_{t}+w_{t}$ is invertible. Denote its inverse by $\alpha_{t} \wedge d t+\beta_{t}$, with $\alpha_{t}$ and $\beta_{t}$ forms on $Z$ depending smoothly on $t \in(-\delta, \delta)$. Then $\omega:=\left.\pi\right|_{M \backslash Z} ^{-1}$ can be written as

$$
\left.\omega\right|_{U \backslash Z}=\alpha_{t} \wedge d t / t+\beta_{t} .
$$

Since $\omega$ is closed we get that $\alpha_{0}$ and $\beta_{0}$ are closed, and since $d t \wedge \alpha_{0}+\beta_{0}$ is invertible, it follows that $\alpha_{0} \wedge \beta_{0}^{n-1}$ is a volume form on $Z$. Since $Z$ is compact, this implies that $\beta_{0}^{n-1}$ cannot be exact. We will construct a closed 2-form $\omega^{\prime}$ on $M$ whose pullback to $Z$ is $\beta_{0}$; hence $c:=\left[\omega^{\prime}\right]$ will satisfy the conclusion of the theorem.

Let $\chi:(-\delta, \delta) \rightarrow \mathbb{R}$ be a bump function that takes the value 1 for $|t| \leq$ $\delta / 4$, and 0 for $|t| \geq \delta / 2$. Consider the 2 -form $\omega^{\prime}$ on $M \backslash Z$ that coincides with $\omega$ outside of $U$ and on $U \backslash Z$ it is given by

$$
\left.\omega^{\prime}\right|_{U \backslash Z}=\left(\alpha_{t}-\chi(t) \alpha_{0}\right) \wedge d t / t+\beta_{t}
$$

The form $\omega^{\prime}$ extends smoothly to $Z$, since for $|t| \leq \delta / 4$ it can be written as $\omega^{\prime}=\lambda_{t} \wedge d t+\beta_{t}$, where $\lambda_{t}=\int_{0}^{1} \dot{\alpha}_{t s} d s$, or equivalently $\alpha_{t}=\alpha_{0}+t \lambda_{t}$. So $\omega^{\prime}$ is a closed 2 -form on $M$ whose pullback to $Z$ is $\beta_{0}$; thus $\left[\omega^{\prime}\right]^{n-1} \neq 0$. 
If $M$ is not orientable, consider $p: \widetilde{M} \rightarrow M$ the orientable double cover, and let $\gamma: \widetilde{M} \stackrel{\sim}{\longrightarrow}$ be the corresponding deck transformation. We first construct a tubular neighborhood $(\widetilde{r}, t): \widetilde{U} \stackrel{\sim}{\longrightarrow} \times(-\delta, \delta)$ of the singular locus $\widetilde{Z}:=p^{-1}(Z)$ of $\widetilde{\pi}:=p^{*}(\pi)$, with $\widetilde{U}=p^{-1}(U)$, and such that the action of $\gamma$ corresponds to $\gamma(z, t)=(\gamma(z),-t)$, for $(z, t) \in \widetilde{Z} \times(-\delta, \delta)$. The map $\widetilde{r}: \widetilde{U} \rightarrow$ $\widetilde{Z}$ can be constructed by lifting a retraction $r: U \rightarrow Z$. Consider a volume form $\mu_{0}$, and denote by $f$ the smooth function satisfying $\gamma^{*}\left(\mu_{0}\right)=-e^{f} \mu_{0}$. Then the volume form $\mu:=e^{f / 2} \mu_{0}$ satisfies $\gamma^{*}(\mu)=-\mu$. Thus, by shrinking $U$, we can use $t:=\left\langle\widetilde{\pi}^{n}, \mu\right\rangle$ to construct the desired tubular neighborhood. As before, on $\widetilde{Z} \times(-\delta, \delta)$ we can write $p^{*}\left(\left.\omega\right|_{U \backslash Z}\right)=\alpha_{t} \wedge d t / t+\beta_{t}$. Invariance under $\gamma$ implies that $\left(\left.\gamma\right|_{\widetilde{Z}}\right)^{*}\left(\alpha_{t}\right)=\alpha_{-t}$ and $\left(\left.\gamma\right|_{\widetilde{Z}}\right)^{*}\left(\beta_{t}\right)=\beta_{-t}$. In particular $\alpha_{0}$ and $\beta_{0}$ are invariant. Thus, choosing the function $\chi(t)$ from the construction from the orientable case to satisfy $\chi(t)=\chi(-t)$, we obtain an invariant closed 2-form $\omega^{\prime}$ on $\widetilde{M}$ that satisfies $\left[\omega^{\prime}\right]^{n-1} \neq 0$. Invariance implies that $\omega^{\prime}=p^{*}\left(\omega^{\prime \prime}\right)$ for a closed 2-form $\omega^{\prime \prime}$ on $M$; hence $c:=\left[\omega^{\prime \prime}\right]$ satisfies the conclusion.

Remark. Observe that for $Z \neq \emptyset$ the proof of the theorem uses only the compactness of $Z$ and not that of $M$.

\section{References}

[1] R. Goto, Rozansky-Witten invariants of log symplectic manifolds, in 'Integrable systems, topology, and physics (Tokyo, 2000)', 69-84, Contemp. Math., 309, Amer. Math. Soc., Providence, RI, 2002.

[2] M. Gualtieri and S. Li, Symplectic groupoids of log symplectic manifolds, 2012, arXiv:1206.3674v2, to appear in IMRN.

[3] V. Guillemin, E. Miranda and A.R. Pires, Symplectic and Poisson geometry on b-manifolds, 2012; arXiv:1206.2020v1.

[4] R. Nest and B. Tsygan, Formal deformations of symplectic manifolds with boundary, J. Reine Angew. Math. 481 (1996), 27-54.

[5] O. Radko, A classification of topologically stable Poisson structures on a compact oriented surface, J. Symplectic Geom., 1(3) (2002), 523-542.

Department of Mathematics, Utrecht University

3508 TA UTRECHT

The Netherlands

E-mail address: i.t.marcut@uu.nl 
Department of Mathematics, Utrecht University

3508 TA, Utrecht, The Netherlands

E-mail address: b.osornotorres@uu.nl

Received 03/28/2013, accepted 02/26/2014

We would like to thank M. Crainic for useful discussions. The first author was supported by the ERC Starting Grant no. 279729 and the second by the NWO VIDI project "Poisson Topology" no. 639.032.712. 